\title{
DIRECT: A System for Evaluating Information Access Components of Digital Libraries ${ }^{\star}$
}

\author{
Giorgio Maria Di Nunzio and Nicola Ferro \\ Department of Information Engineering - University of Padua, \\ Via Gradenigo, 6/b - 35131 Padova - Italy \\ \{dinunzio, ferro\}@dei.unipd.it
}

Digital Library Management Systems (DLMSs) generally manage collections of multi-media digitalized data and include components that perform the storage, access, retrieval, and analysis of the collections of data. Recently, the new trend of DLMS applications is pushing towards a components/services technology which is becoming more and more standardized [1,2. The results of this new orientation are ad-hoc solutions for different components and services of DLMS: the data repository, the data manager, the search and retrieval components, etc. We are particularly interested in the evaluation aspects that range from measuring and quantifying the performances of the information access and extraction components of a DLMS to designing and developing an architecture for a system capable of supporting this kind of evaluation in the context of DLMSs [34].

An innovative system named Distributed Information Retrieval Evaluation Campaign Tool (DIRECT) is being designed and developed with the aim of integrating the activities among the different entities involved in the evaluation of the information access components of a DLMS. Today, this type of evaluation is carried out in important international evaluation forums which bring research groups together, provide them with the means for measuring the performances of their systems, discuss and compare their work [56178. The main aim of DIRECT is to create a unified view of these kind of evaluation forums especially for the management of the results and the analysis of data. We consider this last point (data analysis) a key issue for DIRECT; in fact, since generally evaluation forums do not further the systematical employment of statistical analysis from participants, the important innovative aspect of DIRECT is to provide participants with a uniform way of performing statistical analysis on their results. In this way, not only participants benefit from standard experimental collections, that make the experimental results comparable, but also they may exploit standard tools for the analysis of the experimental results, that make the analysis and assessment of experimental results comparable too. Moreover, giving to participants the possibility to interact with other participants' performances would certainly improve the quality of research.

DIRECT will mainly support the storage and preservation of data together with its retrieval and management. The concept of data in evaluation forums

* The authors wish to thank Luca Pretto and Franco Crivellari for the time spent discussing the entity-relationship schema. The work is partially supported by the DELOS Network of Excellence on Digital Libraries, as part of the Information Society Technologies (IST) Program of the European Commission (Contract G038-507618).

A. Rauber et al. (Eds.): ECDL 2005, LNCS 3652, pp. 483484 2005.

(C) Springer-Verlag Berlin Heidelberg 2005 
may vary from textual documents to statistical analysis of performances, and, in this work, we concentrate mainly on the following aspects:

- the management of an evaluation campaign from the point of view of organizers. The edition, the publication of the act of the workshop, the track set-up, the harvesting of documents;

- the management of users/participants of an evaluation campaign. The registration of the groups, the permission settings;

- the management of pooling assessment. The choice of the assessors, the selection of documents to be assessed, the gathering of assessed documents;

- the management of submission of runs and the validation of submitted runs;

- the management of statistical analysis of participants performances. The choice of the statistical test;

- the management of logs.

The architecture of DIRECT consists of three layers - data, application and interface logic layers - which allow one to achieve a better modularity and to properly describe the behavior of DIRECT by isolating specific functionalities at the proper layer. Moreover, this decomposition makes it possible to clearly define the functioning of DIRECT by means of communication paths that connect the different components. In this way, the behavior of the system is designed in a modular and extensible way.

Since we are going to provide and manage the technical infrastructure, both hardware and software, for the Cross-Language Evaluation Forum (CLEF) 2005 ongoing evaluation campaign, the possibility of testing and evaluating the DIRECT system in real settings will be exploited.

\section{References}

1. DELOS: Newsletter - Issue 2. http://www.delos.info/newsletter/issue2/ [last visited 2004, November 22] (2004)

2. Rauber, A.: DELOS and the Future of Digital Libraries. D-Lib Magazine, 10(10).http://www.dlib.org/dlib/october04/10contents.html [last visited 2004, November 22] (2004)

3. Agosti, M., Di Nunzio, G.M., Ferro, N.: Evaluation of a Digital Library System. In Agosti, M., Fuhr, N., eds.: Notes of the DELOS WP7 Workshop on the Evaluation of Digital Libraries, http://dlib.ionio.gr/wp7/workshop2004_program.html (2004) $73-78$

4. Di Nunzio, G.M., Ferro, N.: DIRECT: a Distributed Tool for Information Retrieval Evaluation Campaigns. In: Proc. 8th International Workshop of the DELOS Network of Excellence on Digital Libraries on Future Digital Library Management Systems (System Architecture \& Information Access). (2005) (in print)

5. Text REtrieval Conference (TREC). (http://trec.nist.gov)

6. Cross Language Evaluation Forum (CLEF). (http://clef.isti.cnr.it)

7. NII-NACSIS Test Collection for IR Systems (NTCIR). (http://research.nii.ac.jp/ntcir/index-en.html)

8. INitiative for the Evaluation of XML Retrieval (INEX). (http://inex.is.informatik.uni-duisburg.de) 